\title{
THE MILNE PROBLEM FOR THE RADIATIVE TRANSFER EQUATIONS (WITH FREQUENCY DEPENDENCE)
}

\author{
FRANÇOIS GOLSE
}

ABSTRACT. We study the following stationary frequency dependent transport equation:

$$
\begin{gathered}
\left.\mu \partial_{x} f+\sigma(\nu, T)\left[f-B_{\nu}(T)\right]=0, \quad x>0, \nu>0, \mu \in\right]-1 ; 1[ \\
\iint_{\mathbf{R}+\times]-1 ; 1]} \sigma(\nu, T)\left[B_{\nu}(T)-f\right] d \nu \frac{d \mu}{2}=0, \\
f(0, \mu, \nu)=\varphi(\mu, \nu), \quad \nu>0, u \in] 0 ; 1[
\end{gathered}
$$

where $B_{\nu}$ is the well-known Planck function appearing in astrophysics. We are able to describe the asymptotic behavior of $f$ and $T$ for $x$ large, when $\sigma(\nu, T)$ is of the special form $\sigma(\nu, T)=\sigma(\nu) k(T)$. Our method relies mainly on the monotonicity of the nonlinearity. The proof does not use any linearization of the equation; in particular, no smallness assumption on the data $\varphi$ (in any sense) is required.

RÉSUMÉ. Nous étudions l'équation de transport stationnaire avec dépendance en fréquence:

$$
\begin{gathered}
\left.\mu \partial_{x} f+\sigma(\nu, T)\left[f-B_{\nu}(T)\right]=0, \quad x>0, \nu>0, \mu \in\right]-1 ; 1[, \\
\iint_{\left.\mathbf{R}^{+} \times\right]-1 ; 1[} \sigma(\nu, T)\left[B_{\nu}(T)-f\right] d \nu \frac{d \mu}{2}=0, \\
f(0, \mu, \nu)=\varphi(\mu, \nu) ; \quad \nu>0, \mu \in] 0 ; 1[.
\end{gathered}
$$

Lorsque $\sigma(\nu, T)$ est de la forme particulière $\sigma(\nu, T)=\sigma(\nu) k(T)$, nous savons décrire le comportement asymptotique de $f$ et $T$ pour $x$ grand. Notre méthode repose principalement sur la monotonie de la non-linéarité. La preuve n'utilise aucune linéarisation de l'équation; en particulier, nous n'avons besoin d'aucune hypothèse de petitesse (d'aucune sorte) sur la donnée $\varphi$.

1. Introduction. We are concerned with the following nonlinear frequency dependent, stationary transport equation:

$$
\begin{gathered}
\left.\mu \partial_{x} f+\sigma(\nu, T)\left[f-B_{\nu}(T)\right]=0, \quad \text { for } x>0, \nu>0, \mu \in\right]-1 ; 1[ \\
\iint_{\left.\mathbf{R}^{+\times}\right]-1 ; 1[} \sigma(\nu, T)\left[B_{\nu}(T)-f\right] d \nu \frac{d \mu}{2}=0 \\
f(0, \mu, \nu)=\varphi(\mu, \nu), \quad \nu>0, \mu \in] 0 ; 1[.
\end{gathered}
$$

Received by the editors July 5,1986 .

1980 Mathematics Subject Classification (1985 Revision). Primary 85A25, 82A70, 35F30; Secondary $65 \mathrm{~N} 99$.

Key words and phrases. Radiative transfer, Milne problem, nonlinear transport equations, boundary layers (transfert radiatif, problème de Milne, equations de transport non-linéaires, couches limites).

The author wishes to thank gratefully Professors C. Bardos, B. Perthame and R. Sentis for most valuable discussions on this topic. 
This problem is usually known as the Milne problem - this denomination being actually used for a broader class of half-space problems in transport theory. Equations (1-1) describe a plane parallel stellar atmosphere in local thermodynamical equilibrium. An introduction to this theory can be found in $[\mathbf{1 0}$ or $\mathbf{1 5}]$.

Let us describe the notations in equation (1-1): $x$ denotes the space variable, $x \in \mathbf{R}^{+} ; \nu$ denotes the frequency variable, $\nu \in \mathbf{R}^{+} ; \mu$ denotes the angular variable, $\mu \in[-1 ; 1]$. Indeed, the situation is monodimensional, and $\mu$ denotes the projection of the speed of photons onto the principal direction (once the speed of light in the atmosphere has been normalized to 1). The two unknowns of (1-1) are

$f=f(x, \mu, \nu)$, density probability of photons at position $x$, with velocity described by the parameter $\mu$, and with frequency $\nu$;

$T=T(x)$, proportional to the fourth power of the material temperature, at position $x$.

The function $B_{\nu}$ is known in astrophysics as the Planck function; after a suitable normalization it can be expressed as

$$
B_{\nu}(T)=\left(15 \nu^{3} / \pi^{4}\right)\left[\exp \left(\nu / T^{-1 / 4}\right)-1\right]^{-1}
$$

so that

$$
\int_{\mathbf{R}^{+}} B_{\nu}(T) d \nu=T .
$$

The function $\sigma(\nu, T)$ is the opacity of the matter at temperature given by $T$ for radiations of frequency $\nu$. Very few general informations about $\sigma$ can be obtained; we only can ensure that $\sigma>0$, but, usually, $\sigma$ is strongly oscillating with respect to both $\nu$ and $T$. In a wide range of physical applications, we also have

$$
\text { for a.e. } T>0, \quad \sigma(\nu, T) \sim \nu^{-\beta}
$$

(see the example known as the Kramer opacity described in [2]). Although (1-4) is not essential mathematically speaking, it will require some refinements of the general features of our method to study (1-1).

The results in this paper are valid only for $\sigma(\nu, T)$ of the special form $\sigma(\nu, T)=$ $\sigma(\nu) k(T)$; however, we shall require no monotonicity assumption as in the previous papers on this topic: see $[\mathbf{2}, \mathbf{1 4}, \mathbf{1 7}$ and 19]. In particular, our assumptions will allow $\sigma$ to oscillate with respect to both $\nu$ and $T$.

The main goal of this paper is to prove the following

"THEOREM". Assume that $\sigma(\nu, T)$ is of the form $\sigma(\nu, T)=\sigma(\nu) k(T)$. Then, (1-1) admits a unique bounded solution. When $x$ is going to infinity, this solution converges in some sense to some Planckian state $\left(B_{\nu}(\alpha), \alpha\right)$, with exponential speed if $\inf _{\nu} \sigma(\nu)>0$, and with subexponential speed if $\sigma(\nu)$ satisfies (1-4).

(We use the denomination "exponential speed" if the error term is of order $\exp (-\gamma x)$ (with $\gamma>0$ ), and "subexponential speed" if the error term is of order $\exp \left(-\gamma x^{\alpha}\right)($ with $0<\alpha<1)$.)

To our knowledge, till now, this kind of result on half-space problems has been proved only for linear cases. Several methods have been used: ergodic theory in [5], Wiener-Hopf techniques in [9], and more recently, energy methods in a series of papers: $[\mathbf{1}, \mathbf{2}$, and 3]. In [2], the nonlinear case (1-1) is treated, but only when $\sigma$ is a function of $T$ alone ("grey" problem). This special feature allows a reduction 
to the linear case, by using an implicit change of variables, introducing the optical depth:

$$
\left.\int_{0}^{r} \sigma(T \xi)\right) d \xi
$$

instead of $x$ as the good space variable. Of course, this technique would be totally irrelevant here, since the optical depth depends on the frequency (for the non-grey problem). However, we shall use this change of variables (1-3) at the end of the paper, to reduce the case where $\sigma(\nu, T)=\sigma(\nu) k(T)$ to the case where $\sigma$ is a function of $\nu$ alone.

The linearization of (1-1) around a fixed Planckian state has been studied in [13 and 19]; the asymptotic states for the linearized (1-1) can be computed almost explicitly by following a method initiated by Chandrasekhar (see the chapter in [10] devoted to the $H$-function), and fully developed in [18]. Whether it is possible to consider the linearization of (1-1) around a Planckian state as an approximation of (1-1) will be discussed at the end of the paper. This question is of significant importance as regards the numerical computation of the asymptotic state $\left(B_{\nu}(\alpha), \alpha\right)$ as in the "Theorem". The method we intend to follow in order to prove the "Theorem" is inspired from the one used in [3] for the linear Milne problem:

$$
\begin{cases}\mu \partial_{x} u+L u=0, & x>0, \mu \in[-1 ; 1] \\ u(0, \mu)=\varphi(\mu), & \mu \in] 0 ; 1]\end{cases}
$$

with

$$
(L u)(x, \mu)=u(x, \mu)-\int_{-1}^{1} u\left(x, \mu^{\prime}\right) \frac{d \mu^{\prime}}{2} .
$$

One of the main features of problem (1-1) is the genuinely nonlinear coupling between $\mu, \nu$, and $T$, due to $\sigma(\nu, T)$. Even in the case where $\sigma$ is of the form $\sigma(\nu, T)=\sigma(\nu) k(T)$, this coupling subsists in some sense: the good invariant quantity for bounded solutions is

$$
\int_{-1}^{1} \int_{0}^{\infty} \frac{\mu^{2}}{\sigma(\nu)} f(x, \mu, \nu) d \nu \frac{d \mu}{2}
$$

the analogue for (1-6) being $\int_{-1}^{1} \mu^{2} u(x, \mu) d \mu / 2$; this means that $\nu$ can in no way be regarded as a mere parameter in the transfer equation. Another striking difference between (1-1) and (1-6) is the strong $L^{2}$ coercivity of $L$, when restricted to $(\operatorname{Ker} L)^{\perp}$; there is no equivalent property for (1-1). Yet, beside the change of variable (1-5), the main tool we are going to use is the $L^{1}$ accretiveness of the nonlinear "collision" operator. In particular, the strategy of this paper would have to be modified in order to fit in with other nonlinear Milne problems, as the computation of the Knudsen layers for the Boltzmann equation $[6,7]$ or the interface layers in the semiconductors theory, since the collision operator in both of these cases is by no mean accretive.

Let us indicate another motivation for studying problems like (1-1). When computing radiative transfer phenomena in very opaque media; one is naturally led to a nonlinear form of the diffusion approximation (for linear transport operators, see $[\mathbf{3}, \mathbf{5}, \mathbf{1 1}])$ called the Rosseland approximation. This approximation has been 
described in [12 and 16], and a first rigorous proof of its validity has been given in [2]. Still, as explained in $[\mathbf{2}, \mathbf{1 2}, \mathbf{1 6}]$, this approximation is valid in the interior only. To extend the approximation near the boundary, we are led to study the asymptotic behavior of (1-1). Then it remains to match the interior approximation to the boundary layer. This last problem seems rather technical because of the nonlinearities in the radiative transfer equations, and is not considered in this article.

The outline of the paper is as follows. In $\S 2$, we present a basic review of the prerequisites and previous results useful for our purposes. In $\S 3$, we give the proof of the exponential decay when $\sigma(\nu, T)=\sigma(\nu)>\sigma_{m}>0$. In $\S 4$, we give the proof of the subexponential decay when $\sigma(\nu, T)=\sigma(\nu) \sim \nu^{-\beta}$. In $\S 5$, we extend the result of $\S \S 3$ and 4 to the case where $\sigma(\nu, T)=\sigma(\nu) k(T)$.

In $\S 6$, we consider the possibility of using the linearized version of (1-1) around a Planckian state (as in $[\mathbf{1 3}, \mathbf{1 9}])$ to compute the asymptotic states of (1-1).

In the Appendix, we give an existence and uniqueness result for (1-1), and also a weak asymptotic result, valid for $\sigma(\nu, T)=\sigma(\nu)$. All these results were proved by Sentis for more general opacities; however, Sentis' proof is quite long and technical, and the assumption $\sigma(\nu, T)=\sigma(\nu)$ will allow important reductions, especially for the uniqueness part.

2. Basic review. We consider problem (1-1), where the opacity is of the special form $\sigma(\nu, T)=\sigma(\nu)$ :

(i) $\mu \partial_{x} f+\sigma(\nu)\left[f-B_{\nu}(T)\right]=0, \quad$ for $\left.x>0, \nu>0, \mu \in\right]-1 ; 1[$;

(ii) $\left\langle\left\langle\sigma(\nu)\left[B_{\nu}(T)-f\right]\right\rangle\right\rangle=0$;

(iii) $f(0, \mu, \nu)=\varphi(\mu, \nu), \quad \nu>0, \mu \in] 0 ; 1[$;

with the following notations which will be in use throughout this paper:

$$
\begin{aligned}
\langle\langle g\rangle\rangle= & \int_{-1}^{1} \int_{0}^{\infty} g(\mu, \nu) d \nu \frac{d \mu}{2}, \quad \text { for } g \in L^{1}(]-1 ; 1\left[\times \mathbf{R}^{+}\right) ; \\
& V=L^{1}(]-1 ; 1\left[\times \mathbf{R}^{+}\right) \cap L^{\infty}(]-1 ; 1\left[\times \mathbf{R}^{+}\right) .
\end{aligned}
$$

Let us now recall the results of Sentis for this problem (see [19]). In this section, we shall need the following assumptions on $\sigma$ :

$$
\sigma>0, \sigma \in L^{\infty}\left(\mathbf{R}^{+}\right) \text {, and for any } \theta>0, B_{\nu}(\theta) / \sigma(\nu) \in L^{1}\left(\mathbf{R}^{+}\right) .
$$

We begin with the following lemma which asserts that (2-2) defines $T$ as nonlinear nondecreasing function of $f$ :

LEMMA 1. For any $u \in L^{1}(]-1 ; 1\left[\times \mathbf{R}^{+}\right)^{+}$, and under assumptions (2-1), there exists a unique nonnegative constant $T_{u}$ such that $\left\langle\left\langle\sigma(\nu)\left[B_{\nu}\left(T_{u}\right)-u\right]\right\rangle\right\rangle=0$. The mapping $u \rightarrow T_{u}$ is continuous and strictly increasing on $L^{1}(]-1 ; 1\left[\times \mathbf{R}^{+}\right)^{+}$.

In view of Lemma 1 , we introduce the following nonlinear operator on $L^{1}\left(\left[-1 ; 1\left[\times \mathbf{R}^{+}\right)\right.\right.$:

$$
Q u=\sigma(\nu)\left[u-B_{\nu}\left(T_{u}\right)\right], \quad \text { with domain } D(Q)=L^{1}(]-1 ; 1\left[\times \mathbf{R}^{+}\right)^{+} .
$$

$Q$ is continuous from $D(Q)$ into $L^{1}(]-1 ; 1\left[\times \mathbf{R}^{+}\right)$, and we have obviously the following property:

$$
Q u=0 \Leftrightarrow \text { there exists } \alpha>0 \text { such that } u=B_{\nu}(\alpha) .
$$


Therefore, problem (2-1) may be rewritten as

$$
\begin{aligned}
& \text { (i) } \mu \partial_{x} f+Q f=0, \\
& \text { (ii) } T(x)=T_{f(x, . . .)}, \quad x>0, \\
& \text { (iii) } f(0, \mu, \nu), \quad \nu>0, \mu \in] 0 ; 1[.
\end{aligned}
$$

The results of Sentis on (2-1) are summarized in the following theorem:

THEOREM 2 [19]. We make assumption (2-2).

(a) (existence and uniqueness): Assume that $0 \leq \psi \leq B_{\nu}(\theta)$; then there exists a unique solution of $(2-1),(f, T)$, in the class $L^{\infty}\left(\mathbf{R}^{+} ; V\right)^{+} \cap L^{\infty}\left(\mathbf{R}^{+}\right)^{+}$.

(b) (maximum-minimum principle): If $\varphi \in V^{+}$satisfies

$$
\varphi \leq B_{\nu}(\theta) \quad\left(\text { resp. } \varphi \geq B_{\nu}(\theta)\right), \quad \text { a.e. in } \mu \text { and } \nu,
$$

then the bounded solution of $(2-1),(f, T)$, satisfies

$$
T \leq \theta \quad(\text { resp. } T \geq \theta) \quad \text { a.e. in } x
$$

and

$$
f \leq B_{\nu}(\theta) \quad\left(\text { resp. } f \geq B_{\nu}(\theta)\right) \quad \text { a.e. in } x, \mu, \nu .
$$

(c) ("weak" asymptotic behavior): Let $(f, T)$ be a bounded solution of (2-1) then there exists $\alpha \geq 0$, and a sequence $x_{n} \rightarrow+\infty$, such that

$$
T\left(x_{n}\right) \rightarrow \alpha \quad \text { and } f\left(x_{n}, ., .\right) \rightarrow B_{\nu}(\alpha)
$$

a.e. in $\mu$ and $\nu$, and in $L^{1}(]-1 ; 1\left[\times \mathbf{R}^{+}\right)$.

REMARK. Uniqueness holds only in the class of bounded functions; even for the linear half-space problems considered in [3 and 5], there exist several unbounded solutions of the same equation. For example, $u(x, \mu)=-3 F(x-\mu)$ is a solution of (1-6) with macroscopic flux

$$
F=\int_{-1}^{1} \mu u(x, \mu) \frac{d \mu}{2} .
$$

As well as for (1-6), bounded solutions of (1-1) (with $\sigma$ satisfying the monotonicity assumptions of [19]) are of macroscopic flux equal to zero. However, this is not a common feature of the Milne problems; the one considered in [1] (for the linearized Boltzmann equation) is of slightly different structure. Because of the special form of the opacity considered in this paper $(\sigma(\nu, T)=\sigma(\nu) k(T)),(2-1)$ admits an additional invariant macroscopic quantity.

PROPOSITION 3 (INVARIANT QUANTITIES). If $f \in L^{\infty}\left(\mathbf{R}^{+} ; V\right)^{+}$satisfies (2-5)(i)(iii) in the sense of distributions, then we have

$$
\begin{aligned}
& \text { (i) }\langle\langle\mu f\rangle\rangle(x)=0, \text { for } x>0, \\
& \text { (ii) }\left\langle\left\langle\left(\mu^{2} / \sigma\right) f\right\rangle\right\rangle(x)=C, \text { for } x>0
\end{aligned}
$$

(where $C$ is a positive constant).

PROOF OF PROPOSITION 3. Integrating (2-1) with respect to $\mu$ and $\nu$, and taking (2-1)(ii) into consideration yields

$$
d_{x}\langle\langle\mu f\rangle\rangle=0, \quad \text { i.e. } \quad\langle\langle\mu f\rangle\rangle(x)=C_{1}
$$


for some constant $C_{1}$. Then, we multiply (2-1)(i) by $\mu / \sigma$, and we integrate it with respect to $\mu$ and $\nu$ to obtain

$$
d_{x}\left\langle\left\langle\left(\mu^{2} / \sigma\right) f\right\rangle\right\rangle=-C_{1}, \quad \text { i.e. } \quad\left\langle\left\langle\left(\mu^{2} / \sigma\right) f\right\rangle\right\rangle(x)=C-C_{1} x
$$

for some constant $C$. And since $f$ is in $L^{\infty}\left(\mathbf{R}^{+} ; V\right)$, this last relation ensures that $C_{1}=0$.

For the proof of Theorem 2, we refer to the Appendix.

3. The case where $\sigma(\nu, T)=\sigma(\nu) \geq \sigma_{m}>0$ In this section, we are concerned with problem (2-1), where the opacity satisfies the following hypothesis:

there exist two constants $0<\sigma_{m}<\sigma_{M}<+\infty$

such that, for a.e. $\nu>0, \sigma_{m} \leq \sigma(\nu) \leq \sigma_{M}$.

Moreover, we assume that

$$
\begin{aligned}
& \text { there exists } 0<\theta_{M}<+\infty \\
& \text { such that } 0 \leq \varphi(\mu, \nu) \leq B_{\nu}\left(\theta_{M}\right) \text {, a.e. in } \mu \text { and } \nu .
\end{aligned}
$$

Therefore, we may use Theorem 2, and, until the end of this section, $(f, T)$ will be the bounded solution of $(3-1)$, and $\left(B_{\nu}(\alpha), \alpha\right)$ the weak asymptotic value of $(f, T)$ predicted by Theorem 2(c). Proposition 3 can be rewritten as follows:

$$
\text { for any } \theta \geq 0 \text {, we have }
$$

$$
\begin{aligned}
& \text { (i) }\left\langle\left\langle\mu\left(f-B_{\nu}(\theta)\right)\right\rangle\right\rangle=0, \\
& \text { (ii) }\left\langle\left\langle\left(\mu^{2} / \sigma(\nu)\right)\left[f-B_{\nu}(\theta)\right]\right\rangle\right\rangle=\left\langle\left\langle\left(B_{\nu}(\alpha)-\left(B_{\nu}(\theta)\right) / 3 \sigma\right\rangle\right\rangle .\right.
\end{aligned}
$$

Now, we state the main result of this section.

THEOREM 4. Under assumptions (3-1)--(3-2), we have

(a) $\left\|f-B_{\nu}(\alpha)\right\|_{L^{1}(\mu, \nu)} \leq C e^{-\gamma x}$,

(b) $|T(x)-\alpha| \leq C^{\prime} e^{-\gamma x}$,

where $0<\gamma<\sigma_{m} / 3$, and $C=C\left(\theta_{M}, \sigma_{M}, \sigma_{m}, \gamma\right)$ and $C^{\prime}=C^{\prime}\left(\theta_{M}, \sigma_{M}, \sigma_{m}, \gamma\right)$ are two positive constants.

From now on, we introduce the following notation which will be of constant use in the sequel:

$$
\begin{aligned}
& \Psi_{f, g}=\operatorname{sgn}^{+}(f-g)-\operatorname{sgn}^{+}\left(T_{f}-T_{g}\right), \quad \text { for any }(f, g) \in V^{+} \times V^{+}, \\
& \psi_{f, \theta}=\Psi_{f, B_{\nu}(\theta)} .
\end{aligned}
$$

We begin the proof with the following lemma which states, roughly speaking, that $Q$ is $T$-accretive (see [4 and 14]); in fact the following result is a little more explicit:

LEMMA 5. For any $(f, g) \in V^{+} \times V^{+}$, we have

(3-5) $(f-g) \Psi_{f, g} \leq 0, \quad$ and $\left[B_{\nu}\left(T_{f}\right)-B_{\nu}\left(T_{g}\right)\right]\left(-\Psi_{f, g}\right) \geq 0, \quad$ a.e. in $\mu$ and $\nu$.

Therefore the operator $Q$ is T-accretive since we have

$$
\begin{aligned}
& \left\langle\left\langle(Q f-Q g) \operatorname{sgn}^{+}(f-g)\right\rangle\right\rangle \\
& \quad=\left\langle\left\langle\sigma(\nu)(f-g) \Psi_{f, g}\right\rangle\right\rangle+\left\langle\left\langle\sigma(\nu)\left[B_{\nu}\left(T_{f}\right)-B_{\nu}\left(T_{g}\right)\right]\left(-\Psi_{f, g}\right)\right\rangle\right\rangle \leq 0 .
\end{aligned}
$$

Moreover, if $\langle\langle\mu(f-g)\rangle\rangle=0$, then we also have the following formula:

$$
\begin{aligned}
& \left\langle\left\langle\mu\left[(f-g)-\left(B_{\nu}\left(T_{f}\right)-B_{\nu}\left(T_{g}\right)\right)\right] \operatorname{sgn}^{+}(f-g)\right\rangle\right\rangle \\
& \quad=\left\langle\left\langle\mu(f-g) \Psi_{f, g}\right\rangle\right\rangle+\left\langle\left\langle\mu\left[B_{\nu}\left(T_{f}\right)-B_{\nu}\left(T_{g}\right)\right]\left(-\Psi_{f, g}\right)\right\rangle\right\rangle,
\end{aligned}
$$


Proof OF LEMMA 5. We have

$$
\begin{aligned}
\langle\langle(Q u) & \left.\left.\cdot \operatorname{sgn}^{+}\left(u-B_{\nu}(\theta)\right)\right\rangle\right\rangle \\
= & \left\langle\left\langle\sigma(\nu)\left(u-B_{\nu}(\theta)\right)^{+}\right\rangle\right\rangle \\
& -\left\langle\left\langle\sigma(\nu)\left(B_{\nu}\left(T_{u}\right)-B_{\nu}(\theta)\right) \operatorname{sgn}^{+}\left(u-B_{\nu}(\theta)\right)\right\rangle\right\rangle .
\end{aligned}
$$

According to (3-1)(ii) and to the fact that $\operatorname{sgn}^{+}\left(T_{u}-\theta\right)$ does not depend on $\mu$ and $\nu$, we have

$$
\begin{aligned}
& \left\langle\left\langle\sigma(\nu)\left(B_{\nu}\left(T_{u}\right)-B_{\nu}(\theta)\right) \operatorname{sgn}^{+}\left(T_{u}-\theta\right)\right\rangle\right\rangle \\
& \quad+\left\langle\left(\sigma(\nu)\left(u-B_{\nu}(\theta)\right)\left(-\operatorname{sgn}^{+}\left(T_{u}-\theta\right)\right)\right\rangle\right\rangle=0 .
\end{aligned}
$$

By adding $(\alpha)$ to $(\beta)$, and assuming (3-5) to be true, we obtain (3-6). Then, if $\langle\langle\mu(f-g)\rangle\rangle=0$, we obtain (3-7) in the same way. It remains to prove (3-5). But, we have that

$$
x y \leq x^{+} \quad\left(=x \cdot \operatorname{sgn}^{+}(x)\right), \quad \text { whenever } x \in \mathbf{R} \text { and } 0 \leq y \leq 1 .
$$

According to this remark, the first inequality in (3-5) is obvious; to obtain the second one, we need the following supplementary remark: since $B_{\nu}$ is increasing for $\nu>0$, we have

$$
\operatorname{sgn}^{+}\left[B_{\nu}\left(T_{u}\right)-B_{\nu}(\theta)\right]=\operatorname{sgn}^{+}\left(T_{u}-\theta\right) .
$$

REMARK. We can see from the result of Lemma 5 how weakly the operator $Q$ is coercive. Both of the terms in the right-hand side of (3-9) only force $u$ and $T_{u}$ to be on the same side of $B_{\nu}(\theta)$ and $\theta$ respectively. This is in strong contrast with the linear cases considered in [1 and 2], where the strong coerciveness of the analogue of operator $Q$ is essential to obtain the exponential decay: for the example (1-6), $L$ is strongly coercive when restricted to $(\operatorname{Ker} L)^{\perp}$. In the present case, we must follow another route, namely to obtain exponential decay on enough moments of the solution.

PROOF OF THEOREM 3. We divide this proof into three steps. We begin with

Step 1. Decay for the term $\left\langle\left\langle\mu\left(f-B_{\nu}(\theta)\right)^{+}\right\rangle\right\rangle$. We multiply property (2-1)(i) by $\operatorname{sgn}^{+}\left(f-B_{\nu}(\theta)\right)$, where $\theta \geq 0$, and use (3-6). By integrating with respect to $\mu$ and $\nu$, we obtain

$$
d_{x}\left\langle\left\langle\mu\left(f-B_{\nu}(\theta)\right)^{+}\right\rangle\right\rangle=-\left\langle\left\langle(Q f) \cdot \operatorname{sgn}^{+}\left(f-B_{\nu}(\theta)\right)\right\rangle\right\rangle \leq 0 .
$$

Thus, $\left\langle\left\langle\mu\left(f-B_{\nu}(\theta)\right)^{+}\right\rangle\right\rangle(x)$ is a decreasing function of $x$. Moreover, according to Theorem 2(c)

$$
\left\langle\left\langle\mu\left(f-B_{\nu}(\theta)\right)^{+}\right\rangle\right\rangle\left(x_{n}\right) \rightarrow\left\langle\left\langle\mu\left(B_{\nu}(\alpha)-B_{\nu}(\theta)\right)^{+}\right\rangle\right\rangle=0 .
$$

Therefore, we have

$$
\left\langle\left\langle\mu\left(f-B_{\nu}(\theta)\right)^{+}\right\rangle\right\rangle \geq 0 .
$$

Then we multiply (3-1)(i) by $\operatorname{sgn}^{+}\left(f-B_{\nu}(\theta)\right) e^{\delta x}$, and use (3-4) again. By integrating with respect to $\mu$ and $\nu$, we obtain

$$
\begin{aligned}
& d_{x}\left[\left\langle\left\langle\mu\left(f-B_{\nu}(\theta)\right)^{+}\right\rangle\right\rangle e^{\delta x}\right]-\delta e^{\delta x}\left\langle\left\langle\mu\left(f-B_{\nu}(\theta)\right)^{+}\right\rangle\right\rangle \\
& \quad+\left\langle\left\langle\sigma(\nu)\left(f-B_{\nu}(\theta)\right) \psi_{f, \theta}\right\rangle\right\rangle e^{\delta x} \\
& \quad+\left\langle\left\langle\sigma(\nu)\left(B_{\nu}\left(T_{f}\right)-B_{\nu}(\theta)\right)\left(-\psi_{f, \theta}\right)\right\rangle\right\rangle e^{\delta x}=0 .
\end{aligned}
$$


According to (3-3)(i) and to (3-5), we may write

$$
\left\langle\left\langle\mu\left(f-B_{\nu}(\theta)\right)^{+}\right\rangle\right\rangle=\left\langle\left\langle\mu\left(f-B_{\nu}(\theta)\right) \psi_{f, \theta}\right\rangle\right\rangle \leq\left\langle\left\langle\left(f-B_{\nu}(\theta)\right) \psi_{f, \theta}\right\rangle\right\rangle .
$$

By using (3-10) and integrating (3-9) on $[0 ; X]$, we obtain

$$
\begin{aligned}
\langle\langle\mu(f- & \left.\left.\left.B_{\mu}(\theta)\right)^{+}\right\rangle\right\rangle(X) e^{\delta X}+\int_{0}^{x}\left(\sigma_{m}-\delta\right)\left\langle\left\langle\left(f-B_{\nu}(\theta)\right) \psi_{f, \theta}\right\rangle\right\rangle e^{\delta \xi} d \xi \\
& +\int_{0}^{x}\left\langle\left\langle\sigma(\nu)\left(B_{\nu}\left(T_{f}\right)-B_{\nu}(\theta)\right)\left(-\psi_{f, \theta}\right)\right\rangle\right\rangle e^{\delta \xi} d \xi \\
\leq & \left\langle\left\langle\mu\left(f-B_{\nu}(\theta)\right)^{+}\right\rangle\right\rangle(0) \leq \theta_{M} .
\end{aligned}
$$

Now, we choose $0<\delta<\sigma_{m}$; (3-11) gives the following conclusions:

$$
\begin{aligned}
& \text { (i) } \begin{array}{l}
\left.\left\langle\mu\left(f-B_{\nu}(\theta)\right)^{+}\right\rangle\right\rangle(X) \leq \theta_{M}, \\
\text { (ii) } \int_{0}^{x}\left\langle\left\langle\left(f-B_{\nu}(\theta)\right)\left[\operatorname{sgn}^{+}\left(f-B_{\nu}(\theta)\right)-\operatorname{sgn}^{+}\left(T_{f}-\theta\right)\right]\right\rangle\right\rangle e^{\delta \xi} d \xi \\
\quad \leq \theta_{M} /\left(\sigma_{m}-\delta\right), \\
\text { (iii) } \int_{0}^{x}\left\langle\left\langle\sigma(\nu)\left(B_{\nu}\left(T_{f}\right)-B_{\nu}(\theta)\right)\right.\right. \\
\left.\left.\quad \times\left[\operatorname{sgn}^{+}\left(T_{f}-\theta\right)-\operatorname{sgn}^{+}\left(f-B_{\nu}(\theta)\right)\right]\right\rangle\right\rangle e^{\delta \xi} \xi \leq \theta_{M} .
\end{array}
\end{aligned}
$$

Now, we are going to use the results in (3-12) to obtain exponential decay on the second moment:

$$
\left\langle\left\langle\left(\nu^{2} / \sigma(\nu)\right)\left[f-B_{\nu}(\alpha)\right]^{+}\right\rangle\right\rangle .
$$

Step 2. Decay for the term $\left\langle\left\langle\left(\mu^{2} / \sigma(\nu)\right)\left[f-B_{\nu}(\alpha)\right]^{+}\right\rangle\right\rangle$. Multiplying (3-1)(i) by $\mu / \sigma(\nu)$, we obtain

$$
\left(\mu^{2} / \sigma(\nu)\right) \partial_{x} f+\mu\left[f-B_{\nu}\left(T_{f}\right)\right]=0 .
$$

Then, we multiply (3-13) by $\operatorname{sgn}^{+}\left[f-B_{\nu}(\alpha)\right] e^{\delta x}$, and we integrate it with respect to $\mu$ and $\nu$ to obtain

$$
\begin{aligned}
& d_{x}\left[\left\langle\left\langle\left(\mu^{2} / \sigma(\nu)\right)\left[f-B_{\nu}(\alpha)\right]^{+}\right\rangle\right\rangle e^{\delta x}\right]-\delta e^{\delta x}\left\langle\left\langle\left(\mu^{2} / \sigma(\nu)\right)\left[f-B_{\nu}(\alpha)\right]^{+}\right\rangle\right\rangle \\
& \quad+\left\langle\left\langle\mu\left(f-B_{\nu}(\alpha)\right) \psi_{f, \alpha}\right\rangle\right\rangle e^{\delta x}+\left\langle\left\langle\mu\left(B_{\nu}\left(T_{f}\right)-B_{\nu}(\alpha)\right)\left(\psi_{f, \alpha}\right)\right\rangle\right\rangle e^{\delta x}=0 .
\end{aligned}
$$

Now, we make the following remark, which is completely similar to (3-10). According to (3-3)(ii) and (3-10)(i), we have the following inequality:

$$
\begin{aligned}
\left\langle\left\langle\left(\mu^{2} / \sigma(\nu)\right)\left[f-B_{\nu}(\alpha)\right]^{+}\right\rangle\right\rangle & =\left\langle\left\langle\left(\mu^{2} / \sigma(\nu)\right)\left(f-B_{\nu}(\alpha)\right) \psi_{f, \alpha}\right\rangle\right\rangle \\
& \leq\left(1 / \sigma_{m}\right)\left\langle\left\langle\left(f-B_{\nu}(\alpha)\right) \psi_{f, \alpha}\right\rangle\right\rangle .
\end{aligned}
$$

Then, according to (3-5) and (3-12)

$$
\left|\int_{0}^{x}\left\langle\left\langle\mu\left(f-B_{\nu}(\alpha)\right) \psi_{f, \alpha}\right\rangle\right) e^{\delta \xi} d \xi\right| \leq \theta_{M} /\left(\sigma_{m}-\delta\right)
$$

and

$$
\mid \int_{0}^{x}\left\langle\left\langle\mu\left(B_{\mu}\left(T_{f}\right)-\beta_{\nu}(\alpha)\right)\left(\psi_{f, \alpha}\right)\right\rangle e^{\delta \xi} d \xi\right| \leq \theta_{M} / \sigma_{m}
$$


Moreover, taking (3-15) into consideration, and integrating (3-14) with respect to $x$, we obtain

$$
\begin{aligned}
& \left\langle\left\langle\frac{\mu^{2}}{\sigma(\nu)}\left[f-B_{\nu}(\alpha)\right]^{+}\right\rangle\right\rangle(X) e^{\delta X} \\
& \quad-\frac{\delta}{\sigma_{m}} \int_{0}^{x}\left\langle\left\langle\left(f-B_{\nu}(\alpha)\right) \psi_{f, \alpha}\right\rangle\right) e^{\delta \xi} d \xi \\
& \quad+\int_{0}^{x}\left\langle\left\langle\mu\left(f-B_{\nu}(\alpha)\right) \psi_{f, \alpha}\right\rangle\right\rangle e^{\delta \xi} d \xi \\
& \quad+\int_{0}^{x}\left\langle\left\langle\mu\left(B_{\nu}\left(T_{f}\right)-B_{\nu}(\alpha)\right)\left(-\psi_{f, \alpha}\right)\right\rangle\right\rangle e^{\delta \xi} d \xi \\
& \leq\left\langle\left\langle\left(\mu^{2} / \sigma(\nu)\right)\left[f-B_{\nu}(\alpha)\right]^{+}\right\rangle\right\rangle(0) \leq \theta_{M} / \sigma_{m} .
\end{aligned}
$$

According to the three inequalities above, we obtain

$$
\left\langle\left\langle\left(\mu^{2} / \sigma(\nu)\right)\left[f-B_{\nu}(\alpha)^{+}\right\rangle\right\rangle(X) \leq\left(\theta_{M} / \sigma_{m}\right)\left[3+2 \delta /\left(\sigma_{m}-\delta\right)\right] e^{-\delta X} .\right.
$$

We claim that now, we have enough information to obtain the exponential decay as predicted by Theorem 3 .

Step 3. End of the proof. First, notice that, according to (3-3)(ii) and (3-17), we have

$$
\begin{aligned}
\left\langle\left\langle\left(\mu^{2} / \sigma(\nu)\right)\left|f-B_{\nu}(\alpha)\right|\right\rangle\right\rangle(X) & =2\left\langle\left\langle\left(\mu^{2} / \sigma(\nu)\right)\left[f-B_{\nu}(\alpha)\right]^{+}\right\rangle\right\rangle(X) \\
& \leq\left(\theta_{M} / \sigma_{m}\right)\left[3+2 \delta /\left(\sigma_{m}-\delta\right)\right] e^{-\delta X} .
\end{aligned}
$$

Now, we turn to prove Theorem 4(a). We remark that, for $0<\varepsilon<1$, we have

$$
\begin{aligned}
\left\langle\left\langle\left|f-B_{\nu}(\alpha)\right|\right\rangle\right\rangle(X)= & \int_{|\mu|>\varepsilon} \int_{0}^{+\infty}\left|f-B_{\nu}(\alpha)\right| d \nu \frac{d \mu}{2} \\
& +\int_{|\mu|<\varepsilon} \int_{0}^{+\infty}\left|f-B_{\nu}(\alpha)\right| d \nu \frac{d \mu}{2} \\
\leq & \frac{\sigma_{M}}{\varepsilon^{2}} \int_{|\mu|>\varepsilon} \int_{0}^{+\infty} \frac{\mu^{2}}{\sigma(\nu)}\left|f-B_{\nu}(\alpha)\right| d \nu \frac{d \mu}{2} \\
& +\int_{|\mu|<\varepsilon} \int_{0}^{+\infty}\left|f-B_{\nu}(\alpha)\right| d \nu \frac{d \mu}{2} .
\end{aligned}
$$

Taking into account (3-17) and the maximum principle derived from (3-2), we have

$$
\left\langle\left\langle\left|f-B_{\nu}(\alpha)\right|\right\rangle\right\rangle(X) \leq 2\left(\frac{\sigma_{M} \theta_{M}}{\sigma_{m} \varepsilon^{2}}\right)\left[3+\frac{2 \delta}{\sigma_{m}-\delta}\right] e^{-\delta X}+2 \varepsilon \theta_{M}
$$

for every $0<\varepsilon<1$.

We now take $\varepsilon=e^{\delta X / 3}$ so that we obtain

$$
\left\langle\left\langle\left|f-B_{\nu}(\alpha)\right|\right\rangle\right\rangle(X) \leq 2\left(\frac{\sigma_{M} \theta_{M}}{\sigma_{m}}\right)\left[3+\frac{2 \delta}{\sigma_{m}-\delta} \frac{\sigma_{m}}{\sigma_{M}}\right] e^{-\delta X / 3} .
$$

It is now very easy to obtain Theorem 4(b). Indeed, according to (2-1)(ii), we have

$$
\left\langle\left\langle\sigma(\nu)\left[B_{\nu}\left(T_{f}\right)-B_{\nu}(\alpha)\right]\right\rangle\right\rangle=\left\langle\left\langle\sigma(\nu)\left[f-B_{\nu}(\alpha)\right]\right\rangle\right\rangle ;
$$


multiplying this equality by $\operatorname{sgn}\left(T_{f}-\alpha\right)$ yields

$$
\left\langle\left\langle\sigma(\nu)\left|B_{\nu}\left(T_{f}\right)-B_{\nu}(\alpha)\right|\right\rangle\right\rangle \leq\left\langle\left\langle\sigma(\nu)\left|f-B_{\nu}(\alpha)\right|\right\rangle\right\rangle
$$

and therefore, we have

$$
\begin{aligned}
\left|T_{f}(X)-\alpha\right| & =\left\langle\left\langle\left|B_{\nu}\left(T_{f}\right)-B_{\nu}(\alpha)\right|\right\rangle\right\rangle(X) \leq\left(\sigma_{M} / \sigma_{m}\right)\left\langle\left\langle\left|f-B_{\nu}(\alpha)\right|\right\rangle\right\rangle \\
& \leq \frac{2 \sigma_{M}^{2} \theta_{M}}{\sigma_{m}}\left[1+\frac{1}{\sigma_{M}}+\frac{2}{\sigma_{m}-\delta}\right] e^{-\delta X / 3}
\end{aligned}
$$

The proof of Theorem 4 is now terminated.

REMARK. (1) An estimate on the constants $C$ and $C^{\prime}$, as given in this proof, may be of some interest in the future, to compute effectively the asymptotic state $\left(\alpha, B_{\nu}(\alpha)\right)$.

(2) Even if $\sigma$ was not bounded above, we would have, by using the same method as in Step 3

$$
\left\langle\left\langle\frac{1}{\sigma(\nu)}\left|f-B_{\nu}(\alpha)\right|\right\rangle\right\rangle \leq 2 \theta_{M}\left(1+\frac{1}{\sigma_{m}}+\frac{2}{\sigma_{m}-\delta}\right) e^{-\delta X / 3} .
$$

Even if (3-20) gives no result on the decay of $T_{f}$, we notice that it gives the natural weight for the exponential decay of $f$, namely $1 / \sigma$.

4. The case where $\sigma(\nu, T)=\sigma(\nu) \simeq \sigma_{0} \nu^{-\beta}(\beta>0 ; \nu \rightarrow+\infty)$. In this section, we look at problem (2-1), with the following assumption on the opacity:

there exists three positive constants $\sigma_{0}, \sigma_{M}, \beta$ such that

$$
0<\sigma(\nu)<\sigma_{M} \text {, and } \sigma(\nu) \simeq \sigma_{0} \nu^{-\beta} \text { for } \nu \rightarrow+\infty
$$

$\sigma$ is continuous with respect to $\nu$.

In particular, (4-1) ensures that (2-2) holds, so that the invariant analysis set out in Proposition 3 is still valid. We keep assumption (3-2) on the incoming radiative intensity $\varphi$, and the notations $(f, T)$ and $\left(B_{\nu}(\alpha), \alpha\right)$ respectively for the bounded solution of (2-1) and its weak asymptotic limit predicted by Theorem 2(c). Conclusions (3-3) remain therefore valid. The main result in the present section is:

THEOREM 6. Assume (4-1), (3-2). Therefore, there exists three positive constants $\lambda, K, K^{\prime}$ such that

$$
\left\|f(x, ., .)-B_{\nu}(\alpha)\right\|_{L_{\mu, \nu}}^{1} \leq K e^{-\lambda x^{\rho}}, \quad|T(x)-\alpha| \leq K e^{-\lambda x^{\rho}}, \quad \text { for } x>0 ;
$$

where $\rho=1 /(1+\beta)$.

Before starting the proof of Theorem 6, let us make a few remarks. The introduction of assumption (4-1) was motivated by the existence of opacities vanishing at very high frequencies. We refer to [2], where the example of the so-called Kramer opacity is given:

$$
\sigma(\nu, T)=C\left(1-e^{-\nu / T}\right) /\left(\nu^{3} \sqrt{T}\right) .
$$

Even if Theorem is certainly more pertinent than Theorem 4 in a physical viewpoint, the essential features of the method are already contained in the proof of Theorem 4. In that sense, Theorem 6 is derived from the proof of Theorem 4 in the same spirit as the proof of subexponential decay for the Boltzmann equation with a soft potential (see Caflisch [8]). 
PROOF OF THEOREM 6 . We follow the same route as in the proof of Theorem 4. Since the proof uses a great deal of arguments already contained in the proof of Theorem 4, we are going to point out only the alterations brought by (4-1).

Step 1. Decay for the term $\left\langle\left\langle\mu\left(f-B_{\nu}(\alpha)\right)^{+}\right\rangle\right\rangle$. By proceeding as in the proof of Theorem 4, we easily obtain that (3-8), (3-9), (3-10) are still valid. For $A>0$, let us define $\gamma(A)=\sigma_{0} / 2 A^{\beta}$ and $x(A)=A^{\beta+1} / \sigma_{0} \theta_{M}^{1 / 4}$. According to (4-1), and for $A$ large enough, $\sigma(\nu)>\gamma(A)$, for $\nu \in[0, A]$. By proceeding as in (3-11), we easily obtain, using (3-2) and the maximum principle

$$
\begin{aligned}
\langle\langle\mu(f- & \left.\left.\left.B_{\nu}(\alpha)\right)^{+}\right\rangle\right\rangle(x(A)) e^{\gamma(A) x(A)} \\
& +\int_{0}^{x(A)}\left(\int_{0}^{A} \int_{-1}^{1}(\sigma(\nu)-\gamma(A))\left(f-B_{\nu}(\alpha)\right) \psi_{f, \alpha} \frac{d \mu}{2} d \nu\right) e^{\gamma(A) \xi} d \xi \\
& +\int_{0}^{x(A)}\left\langle\left\langle\sigma(\nu)\left[B_{\nu}\left(T_{f}\right)-B_{\nu}(\alpha)\right]\left(-\psi_{f, \alpha}\right)\right\rangle\right\rangle e^{\gamma(A) \xi} d \xi \\
\leq & C+\int_{0}^{x(A)}\left(\int_{A}^{+\infty} 4 B_{\nu}\left(\theta_{M}\right) \gamma(A) d \nu\right) e^{\gamma(A) \xi} d \xi \\
\leq & C+4 e^{\gamma(A) x(A)} \int_{A}^{+\infty} B_{\nu}\left(\theta_{M}\right) d \nu,
\end{aligned}
$$

where $C$ is positive constant. Now, we have

$$
\int_{A}^{+\infty} B_{\nu}\left(\theta_{M}\right) d \nu \simeq \theta_{M}^{1 / 4} A^{3} \exp \left(\frac{-A}{\theta_{M}{ }^{1 / 4}}\right), \quad \text { for } A \rightarrow+\infty
$$

According to the above definitions of $x(A)$ and $\gamma(A)$, the above inequality yields

$$
\begin{gathered}
\text { (i) }\left\langle\left\langle\mu\left(f-B_{\nu}(\alpha)\right)^{+}\right\rangle\right\rangle(x(A)) e^{\gamma(A) x(A)} \leq C, \\
\text { i.e. }\left\langle\left\langle\mu\left(f-B_{\nu}(\alpha)\right)^{+}\right\rangle\right\rangle(x) \leq C \exp \left[-\left(\sigma_{0} x\right)^{\rho} / 2 \theta_{M}^{\beta \rho / 4}\right] \\
\text { (ii) } \int_{0}^{x(A)}\left(\int_{0}^{A} \int_{-1}^{1}(\sigma(\nu)-\gamma(A))\left(f-B_{\nu}(\alpha)\right) \psi_{f \alpha} \frac{d \mu}{2} d \nu\right) e^{\gamma(A) \xi} d \xi \leq C \text {; } \\
\text { (iii) } \int_{0}^{x(A)}\left\langle\left\langle\sigma(\nu)\left[B_{\nu}\left(T_{f}\right)-B_{\nu}(\alpha)\right]\left(-\psi_{f, \alpha}\right)\right\rangle\right\rangle e^{\gamma(A) \xi} d \xi \leq C .
\end{gathered}
$$

Step 2. Decay for the term $\left\langle\left\langle\left(\mu^{2} / \sigma\right)\left(f-B_{\nu}(\alpha)\right)^{+}\right\rangle\right\rangle$. By proceeding as in the proof of Theorem 4, we easily obtain that (3-13) and (3-14) are still valid. Then we want to write an inequality similar to (3-16) in the proof of Theorem 4 . Taking $\delta=\gamma(A)$, we have

$$
\left\langle\left\langle\left(\mu^{2} / \sigma\right)\left(f-B_{\nu}(\alpha)\right)^{+}\right\rangle\right\rangle x(A / 2) e^{\gamma(A) x(A / 2)}+I_{1}+I_{2}+I_{3} \leq C+I_{4}+I_{5},
$$


where

$$
\begin{aligned}
& I_{1}=\int_{0}^{x(A / 2)}\left\langle\left\langle\mu\left(f-B_{\nu}(\alpha)\right)^{+}\right\rangle\right\rangle(\xi) e^{\gamma(A) \xi} d \xi \\
& I_{2}=\int_{0}^{x(A / 2)}\left(\int_{0}^{A / 2} \int_{-1}^{1}\left[-\gamma(A) \frac{\mu^{2}}{\sigma}\right]\left(f-B_{\nu}(\alpha)\right) \psi_{f, \alpha} \frac{d \mu}{2} d \nu\right) e^{\gamma(A) \xi} d \xi \\
& I_{3}=\int_{0}^{x(A / 2)}\left(\int_{0}^{A / 2} \int_{-1}^{1} \mu\left[B_{\nu}\left(T_{f}\right)-B_{\nu}(\alpha)\right]\left(-\psi_{f, \alpha}\right) \frac{d \mu}{2} d \nu\right) e^{\gamma(A) \xi} d \xi \\
& I_{4}=\int_{0}^{x(A / 2)}\left(\int_{0}^{A / 2} \int_{-1}^{1} \frac{\gamma(A)}{\sigma}\left(f-B_{\nu}(\alpha)\right) \psi_{f, \alpha} \frac{d \mu}{2} d \nu\right) e^{\gamma(A) \xi} d \xi \\
& I_{5}=\int_{0}^{x(A / 2)}\left(\int_{0}^{A / 2} \int_{-1}^{1}\left[B_{\nu}\left(T_{f}\right)-B_{\nu}(\alpha)\right]\left(-\psi_{f, \alpha}\right) \frac{d \mu}{2} d \nu\right) e^{\gamma(A) \xi} d \xi
\end{aligned}
$$

Now, we are going to use the conclusions (4-3) of the previous step to find adequate estimates on the quantities $I_{1}, I_{2}, I_{3}, I_{4}, I_{5}$.

$$
\begin{aligned}
I_{1} & \leq \int_{0}^{x(a / 2)}\left\langle\left\langle\mu\left(f-B_{\nu}(\alpha)\right)^{+}\right\rangle\right\rangle(\xi) e^{\gamma(A / 2) \xi} e^{[\gamma(A)-\gamma(A / 2)] \xi} d \xi \\
& \leq C \int_{0}^{x(A / 2)} e^{[\gamma(A)-\gamma(A / 2)] \xi} d \xi \leq \frac{C}{\gamma(A / 2)} \leq \frac{2 C}{\sigma_{0}}\left(\frac{A}{2}\right)^{\beta},
\end{aligned}
$$

according to $(4-3)(\mathrm{i})$;

$$
\begin{aligned}
I_{2} \leq & \operatorname{Sup}_{0<\nu<A / 2} \frac{\gamma(A)}{\sigma(\nu)(\sigma(\nu)-\gamma(A))} \\
& \cdot \int_{0}^{x(A / 2)}\left(\int_{0}^{A / 2} \int_{-1}^{1}(\sigma(\nu)-\gamma(A))\left(f-B_{\nu}(\alpha)\right) \psi_{f, \alpha} \frac{d \mu}{2} d \nu\right) e^{\gamma(A) \xi} d \xi \leq C \\
\leq & C\left(\operatorname{Sup}_{0<\nu<A / 2} \frac{\gamma(A)}{\sigma(\nu)(\sigma(\nu)-\gamma(A))}\right) \leq \frac{C}{\gamma(A / 2)} \leq \frac{2 C}{\sigma_{0}}\left(\frac{A}{2}\right)^{\beta},
\end{aligned}
$$

according to $(4-3)(\mathrm{ii})$;

$$
\begin{aligned}
I_{3} & \leq \operatorname{Sup}_{0<\nu<A / 2} \frac{1}{\sigma(\nu)} \int_{0}^{x(A / 2)}\left\langle\left\langle\sigma(\nu)\left[B_{\nu}\left(T_{f}\right)-B_{\nu}(\alpha)\right]\left(-\psi_{f, \alpha}\right)\right\rangle\right\rangle e^{\gamma(A) \xi} d \xi \\
& \leq \frac{C}{\gamma(A / 2)} \leq \frac{2 C}{\sigma_{0}}\left(\frac{A}{2}\right)^{\beta}, \quad \text { according to }(4-3)(\mathrm{ii}) ; \\
I_{4} & \leq \int_{0}^{x(A / 2)} e^{\gamma(A) \xi} d \xi \int_{A / 2}^{+\infty} \frac{4 \gamma(A)}{\gamma(A / 2)} B_{\nu}\left(\theta_{M}\right) d \nu \quad \text { (by the maximum principle) } \\
& \leq \frac{e^{\gamma(A) x(A / 2)}}{\gamma(A / 2)} \cdot \int_{A / 2}^{+\infty} 4 B_{\nu}\left(\theta_{M}\right) d \nu \\
& \simeq \frac{4 e^{\gamma(A) x(A / 2)}}{\gamma(A / 2)} \theta_{M}^{1 / 4}(A / 2)^{3} \exp \left(-A / 2 \theta_{M}^{1 / 4}\right), \quad \text { for } A \rightarrow+\infty .
\end{aligned}
$$


According to the choice of the functions $x(A)$ and $\gamma(A)$, we easily obtain

$$
I_{4} \leq \frac{C}{\gamma(A / 2)} \leq \frac{2 C}{\sigma_{0}}\left(\frac{A}{2}\right)^{\beta} .
$$

In the same way

$$
\begin{aligned}
I_{5} & \leq \int_{0}^{x(A / 2)} e^{\gamma(A) \xi} d \xi \int_{A / 2}^{+\infty} 4 B_{\nu}\left(\theta_{M}\right) d \nu \\
& \simeq \frac{4 e^{\gamma(A) x(A / 2)}}{\gamma(A)} \theta_{M}^{1 / 4}(A / 2)^{3} \exp \left(-A / 2 \theta_{M}^{1 / 4}\right), \quad \text { for } A \rightarrow+\infty, \\
& \leq C / \gamma(A) .
\end{aligned}
$$

From (4-4) and the five estimates above, we deduce that

$$
\left\langle\left\langle\left(\mu^{2} / \sigma\right)\left(f-B_{\nu}(\alpha)\right)^{+}\right\rangle\right\rangle(x(A / 2)) e^{\gamma(A) x(A / 2)} \leq C+C A^{\beta},
$$

where $C$ is a positive constant.

Therefore we have

$$
\left\langle\left\langle\left(\mu^{2} / \sigma\right)\left(f-B_{\nu}(\alpha)\right)^{+}\right\rangle\right\rangle(x(A / 2)) \leq C e^{-(\gamma(A) / 2) x(A / 2)} ;
$$

i.e.

$$
\left\langle\left\langle\left(\mu^{2} / \sigma\right)\left(f-B_{\nu}(\alpha)\right)^{+}\right\rangle\right\rangle(X) \leq C \exp \left[-\left(x \sigma_{0}\right)^{\rho} / 2^{\beta+2} \theta_{M}^{\beta \rho / 4}\right] .
$$

As we have pointed out at the beginning of the present section, conclusions (3-3) are not modified by assumption (4-1). Therefore, as in the proof of Theorem 4 (see (3-18)), we have

$$
\begin{aligned}
\left\langle\left\langle\left(\mu^{2} / \sigma\right)\left|f-B_{\nu}(\alpha)\right|\right\rangle\right\rangle(x) & =2\left\langle\left\langle\left(\mu^{2} / \sigma\right)\left(f-B_{\nu}(\alpha)\right)^{+}\right\rangle\right\rangle(x) \\
& \leq C \exp \left[-\left(x \sigma_{0}\right)^{\rho} / 2^{\beta+2} \theta_{M}^{\beta \rho / 4}\right]
\end{aligned}
$$

according to (4-6). From now on, the third step of the proof of Theorem 4 can be applied to the present situation, without any change, so that we obtain

$$
\left\|f(x, ., .)-B_{\nu}(\alpha)\right\|_{L_{\mu, \nu}^{1}} \leq K e^{-a x^{\rho}},
$$

for some positive constants $K$ and $a$. Now, from (4-7) we are going to derive the predicted asymptotic behavior of $T$, as in the proof of Theorem 4, i.e. by using equation (2-1)(ii). We have

$$
\left\langle\left\langle\sigma(\nu)\left(B_{\nu}\left(T_{f}\right)-b_{\nu}(\alpha)\right)\right\rangle\right\rangle(x) \leq \sigma_{M}\left\|f(x, ., .)-B_{\nu}(\alpha)\right\|_{L_{\mu, \nu}^{1}} \leq \sigma_{M} K e^{-a x^{\rho}} .
$$

For $x$ large enough, $\sigma(\nu) \leq \sigma_{0} / 2 x^{\beta}$ for $\nu \in[0, x]$; therefore

From (4-8)

$$
\begin{aligned}
\left\langle\left\langle\left|B_{\nu}\left(T_{f}\right)-B_{\nu}(\alpha)\right|\right\rangle\right\rangle(x) & =\left|T_{f}-\alpha\right|(x) \\
& \leq K \frac{2 \sigma_{M}}{\sigma_{0}} x^{\beta} \exp \left(-a x^{\rho}\right)+\int_{x}^{+\infty} 2 B_{\nu}\left(\theta_{M}\right) d \nu \\
& \simeq K \frac{2 \sigma_{M}}{\sigma_{0}} x^{\beta} \exp \left(-a x^{\rho}\right)+2 \theta_{M}^{1 / 4} x^{3} \exp \left(\frac{-x}{\theta_{M}^{1 / 4}}\right) .
\end{aligned}
$$

$$
\left|T_{f}-\alpha\right|(x) \leq K^{\prime} e^{(-a / 2) x^{\rho}}
$$

so that the decay predicted by Theorem 6 is obtained by taking $\lambda=a / 2$. 
REMARK. For effective numerical computations, problem (2-1) will be descretized by introducting a finite number of groups (in frequency). Therefore, the discretized $\sigma$ will satisfy assumption (3-1), so that the discretized Milne problem will always be ruled by Theorem 4 . This remark is the reason why we have not estimated accurately $K, K^{\prime}$ and $\lambda$ as given by Theorem 6 . These constants will not appear in numerical computations.

5. The case where $\sigma(\nu, T)=\sigma(\nu) k(T)$. In this section, $k$ is a positive function defined on $\mathbf{R}^{+}$such that

there exist 2 constants $0<k_{m}<k_{M}<+\infty$ such that $k_{m}<k(T)<k_{M}$, $k \in C^{1}\left(\mathbf{R}^{+}\right)$.

Now, we assume that the opacity is of the special form: $\sigma(\nu, T)=\sigma(\nu) k(T)$, so that we can rewrite problem (1-1) as:

$$
\begin{aligned}
& \text { (i) } \left.\mu \partial_{x} f+\sigma(\nu) k(T)\left[f-B_{\nu}(T)\right]=0, \text { for } x>0, \nu>0, \mu \in\right]-1 ; 1[\text {, } \\
& \text { (ii) }\left\langle\left\langle\sigma(\nu)\left[B_{\nu}(T)-f\right]\right\rangle\right\rangle=0, \\
& \text { (iii) } f(0, \mu, \nu)=\varphi(\mu, \nu), \quad \nu>0, \mu \in] 0 ; 1[\text {. }
\end{aligned}
$$

In this section, we keep assumption (3-1) or (4-1) on $\sigma$ and definition (2-3). But we can no longer apply Sentis' result $[19]$ because we have no monotonicity assumption on $k$. However, we are going to prove that, in some sense, problems (2-1) and (5-2) are equivalent. Indeed, let $\left(f_{1}, T_{1}\right)$ be the solution of $(2-1)$, and $(f, T)$ be a solution of $(5-2)$; we have the representation formula (at least formally):

$$
f(x, \mu, \nu)=f_{1}\left(\int_{0}^{x} k(T(s)) d s, \mu, \nu\right), \quad T(x)=T_{1}\left(\int_{0}^{x} k(T(s)) d s\right) .
$$

This representation formula will be the key for our proof. Indeed, in view of (5-1), (5-3) gives that $(f, T)$ and $\left(f_{1}, T_{1}\right)$ have the same asymptotic behavior for $x \rightarrow+\infty$.

This same kind of representation formula as (5-3) has already been used in [2] to study the nonlinear Milne problem in the "grey" case (where $\sigma(\nu, T)$ is a function of $T$ only). The argument that will be used here is very much the same as in [2]; it consists in using (5-3) to build a solution of (5-2) with the help of the solution $\left(f_{1}, T_{1}\right)$ of $(2-1)$.

Now, let us state the main result of this section:

THEOREM 7. Under assumptions (5-1), (3-1) or (4-1), and (3-2), there exists a unique bounded solution $(f, T)$ of (5-2). For this solution, the representation formula (5-3) holds. In particular, there exists a Planckian state $\left(\alpha, B_{\nu}(\alpha)\right)$ such that

if (3-1) holds, $\left\|f(x, ., .)-B_{\nu}(\alpha)\right\|_{L^{1}(\mu, \nu)} \leq C e^{-\gamma^{\prime} x}$ and $|T(x)-\alpha| \leq C e^{-\gamma^{\prime} x}$ for $x>0$, where $\gamma^{\prime}=k_{m} \gamma, C$ and $\gamma$ being the same as in Theorem 3 ;

if (4-1) holds, $\left\|f(x, ., .)-B_{\nu}(\alpha)\right\|_{L_{\mu, \nu}^{1}} \leq K e^{-\lambda^{\prime} x^{\rho}}$, and $|T(x)-\alpha| \leq K e^{-\lambda^{\prime} x^{\rho}}$, for $x>0$, where $\lambda^{\prime}=\lambda k_{m}^{\rho}, \lambda, K$, and $\rho$ being the same as in Theorem 6 .

The proof of Theorem 7 follows the same route as in [2] (for the nonlinear Milne problem in the grey case); therefore we are only going to sketch the proof of it. 
PROOF OF THEOREM 7. The representation formula (5-3) gives

$$
k(T(x))=k\left(T_{1}\left(\int_{0}^{x} k(T(s)) d s\right)\right) .
$$

We define $y(x)=\int_{0}^{x} k(T(s)) d s$; so that we have for $y$ the following differential equation:

$$
y^{\prime}(x)=k\left(T_{1}(y(x))\right), \quad y(0)=0 .
$$

Now, we claim that existence and uniqueness for (5-2) is equivalent to existence and uniqueness for the O.D.E. (5-4). Indeed, if $y$ is a solution of (5-4), one easily checks that

$$
f(x, \mu, \nu)=f_{1}(y(x), \mu, \nu), \quad T(x)=T_{1}(y(x))
$$

is a solution of $(5-2)$.

Then, if we have two solutions of $(5-2)$, namely $(f, T)$ and $(\underline{f}, \underline{T})$, let us define

$$
\underline{u}(x)=\int_{0}^{x} k(\underline{T}(s)) d s \quad \text { and } \quad \underline{f}_{1}(y, \mu, \nu)=\underline{f}(\underline{x}(\underline{y}), \mu, \nu), \quad \underline{T}_{1}(y)=\underline{T}(\underline{x}(y))
$$

(where $\underline{x}$ is the reciprocal function of $\underline{u}$, which exists according to (5-1)). We can prove that $\left(\underline{f}, \underline{T}_{1}\right)$ is solution of $(2-1)$, for which we have the uniqueness result of sentis [19] stated here as point (a) in Theorem 2 . Therefore $\underline{f}_{1}=f_{1}$, and $\underline{T}_{1}=T_{1}$, and $y$ is also a solution of (5-4). Moreover,

$$
\underline{f}(x, \mu, \nu)=\underline{f}_{1}(y(x), \mu, \nu), \quad \underline{T}(x)=\underline{T}_{1}(y(x))
$$

so that uniqueness for (5-2) reduces to the uniqueness of the solution of (5-4).

Existence for (5-4). We only need to prove that $T_{1}$ is continuous. According to the results of $\S 5$, we have the following bounds:

$$
\|g\|_{L^{\infty}(x, \mu)} \leq C, \quad\left\|\mu \partial_{x} g\right\|_{L^{\infty}(x, y)} \leq C
$$

where $g(x, \mu)=\int_{0}^{\infty} \sigma(\nu) f_{1}(x, \mu, \nu) d \nu$. Therefore, we may write

$$
\begin{aligned}
& \left|\int_{-1}^{1} g(x, \mu) \frac{d \mu}{2}-\int_{-1}^{1} g(y, \mu) \frac{d \mu}{2}\right| \\
& \quad \leq \int_{|\mu|<\varepsilon}|g(x, \mu)-g(y, \mu)| \frac{d \mu}{2}+\frac{1}{\varepsilon} \int_{|\mu|>\varepsilon}\left|\int_{x}^{y}\right| \mu \partial_{x} g(\xi, \mu)|d \xi| \frac{d \mu}{2} \\
& \quad \leq C+(C / \varepsilon)|x-y| \leq 2 C|x-y|^{1 / 2} .
\end{aligned}
$$

Therefore, $\left\langle\left\langle\sigma(\nu) f_{1}\right\rangle\right\rangle \in C^{1 / 2}\left(\mathbf{R}^{+}\right)$. From (2-1)(ii), we obtain that $T_{1}$ is continuous.

Uniqueness for (5-4). We are going to use a very simple lemma the proof of which can be found in [2].

LEMMA. Let $F \in C_{b}\left(\mathbf{R}^{+}\right), F \geq 1$, and assume that $\left|F^{\prime}(y)\right| \leq \max \left(1 ; y^{-1 / 2}\right)$; then the O.D.E. $y^{\prime}(x)=F(y(x)), y(0)=0$ has a unique solution.

According to assumption (4-1), we only have to prove that

$$
\left|d_{y} T_{1}(y)\right| \leq \max \left(1 ; y^{-1 / 2}\right) \text {. }
$$

We mimic the proof given originally in [3] for an analogous result. We choose for $\left.x_{0} \in\right] 0 ; 1\left[\right.$ a function $\rho \in C^{1}([0,2])$ such that supp $\rho \subset\left[x_{0} / 2 ; 2[, \rho=1\right.$ on 
$\left[x_{0}, 1\right]$, and $\left|d_{x} \rho\right| \leq C / x_{0}$ for some constant $C$ independent of $x$. We introduce $g=\rho \partial_{x} f_{1}, S=\rho d_{x} T_{1}$. We have

$$
\begin{aligned}
L_{T_{1}}(g) & =\mu \partial_{x} g+\sigma(\nu)\left(g-B_{\nu}^{\prime}\left(T_{1}\right)\right)\left\langle\left\langle\sigma(\nu) B_{\nu}^{\prime}\left(T_{1}\right)\right\rangle\right\rangle^{-1}\langle\langle\sigma(\nu) g\rangle\rangle \\
& =-d_{x} \rho \sigma(\nu)\left(f_{1}-B_{\nu}\left(T_{1}\right)\right), \\
S & =\left\langle\left\langle\sigma(\nu) B_{\nu}^{\prime}\left(T_{1}\right)\right\rangle\right\rangle^{-1}\langle\langle\sigma(\nu) g\rangle\rangle, \quad g(0, \mu)=g(2, \mu)=0 .
\end{aligned}
$$

Now, it is fairly classical that 0 does not belong to the spectrum of $L_{T_{1}}$ in

$$
L^{\infty}\left([0 ; 2] \times[-1 ; 1] ; L^{1} \cap L^{\infty}(\nu)\right)
$$

(see [11]). From this remark we deduce very easily that

$$
\begin{aligned}
& \|g\|^{L^{\infty}\left([0 ; 2] \times[-1 ; 1] ; L^{1} \cap L^{\infty}(\nu)\right)} \\
& \quad \leq C\left\|d_{x} \rho \sigma(\nu)\left(f_{1}-B_{\nu}\left(T_{1}\right)\right)\right\|_{L^{\infty}\left([0 ; 2] \times[-1 ; 1] ; L^{1} \cap L^{\infty}(\nu)\right)} \leq C / x_{0} .
\end{aligned}
$$

To prove the estimate on $S$, we write, for $0<x \leq 1$

$$
\begin{gathered}
|\langle\langle\sigma(\nu) g\rangle\rangle| \leq \int_{|\mu|<\left|x_{0}\right|^{1 / 2}} \int_{0}^{\infty}|\sigma(\nu) g| \frac{d \nu}{2} \\
\int_{|\mu|>\left|x_{0}\right|^{1 / 2}} \int_{0}^{\infty} \frac{1}{|\mu|} \mid \sigma(\nu)^{2}\left(f_{1}-B_{\nu}\left(T_{1}\right) \mid d \nu \frac{d \mu}{2} \leq \frac{C}{\left|x_{0}\right|^{1 / 2}} .\right.
\end{gathered}
$$

The remaining details are sheer routine and can be found in [2].

Therefore, we have proved existence and uniqueness of a bounded solution of (4-2), satisfying the representation formula (5-3).

In view of (5-1) and (5-3) the asymptotic behavior of $(f, T)$ is now obvious.

REMARK. The quantity $\int_{0}^{x} k(T(s)) d s$ has a physical meaning; it is called the optical depth.

6. Linearization of (2-1) around a fixed Planckian state. One may think of problem (2-1) as the equation defining the boundary layers arising in the Rosseland approximation (for which we refer to [12 and 16]). Therefore, one may be interested in finding a simple way to compute effectively $\alpha$ (as in Theorem 4 ) in terms of the incoming density $\varphi$, without computing the whole solution of (2-1). For linear Milne problems, such computations are well known, and have inspired much literature. The initial remark was due to Chandrasekhar [10], and a modern interpretation in terms of factorization properties for Riccati equation was given in Sentis [19]. In the same way, one may linearize problem (2-1) around a fixed Planckian state, and, by using Chandrasekhar's techniques, perform the same computation on the linearized problem (2-1): this work has been done by Sentis in [19]. Some improvements can be found in [13]. Now, the question is: can we hope to use Chandrasekhar's techniques on the linearized (2-1) to compute $\alpha$ ?

Assume that we linearize (2-1) around a given Planckian state $\left(\beta, B_{\nu}(\beta)\right)$. Thus we set

Then we have

$$
f=B_{\nu}(\beta)+g ; \quad T=\beta+s .
$$

$$
\begin{aligned}
& \mu \partial_{x} g+\sigma(\nu)\left(g-B_{\nu}^{\prime}(\beta)\left\langle\left\langle\sigma(\nu) B_{\nu}^{\prime}(\beta)\right\rangle\right\rangle^{-1}\langle\langle\sigma(\nu) g\rangle\rangle\right)=O\left(g^{2}\right), \\
& S=\left\langle\left\langle\sigma(\nu) B_{\nu}^{\prime}(\beta)\right\rangle\right\rangle^{-1}\langle\langle\sigma(\nu) g\rangle\rangle, \\
& \left.g(0, \mu, \nu)=\varphi(\mu, \nu)-B_{\nu}(\beta), \quad \nu>0, \mu \in\right] 0 ; 1[.
\end{aligned}
$$


If we drop the inhomogeneity in $O\left(g^{2}\right)$, we can compute easily $\beta$ such that $g \rightarrow 0$ and $S \rightarrow 0$ for $x \rightarrow \infty$, by using Chandrasekhar's techniques [19]. By doing this, we claim that we commit an error of order $O\left[\left(\|g\|_{L^{\infty}}\right)^{2}\right]$; see [5].

Indeed, there is sort of a feedback effect: even if $O\left(g^{2}\right) \rightarrow 0$ exponentially fast for $x$ going to $\infty$, the values of the source term at finite distance perturb the value of $\beta$.

The conclusion is the following: such a computation will be valid only if we know that $\|g\|_{L^{\infty}}$ is small a priori, for example by using the maximum principle on (2-1) if $B_{\nu}\left(\theta_{m}\right) \leq \varphi \leq B_{\nu}\left(\theta_{M}\right)$ with $\theta_{M}-\theta_{m}$ small; in that case we will obtain an approximation of $\alpha$ with error of order $\left(\theta_{M}-\theta_{m}\right)^{2}$.

Appendix. Proof of Theorem 2. This proof is divided into three steps: first, we prove the existence of a solution of (2-1) satisfying the maximum principle; then, we prove that any solution of $(2-1)$ in $L^{\infty}(\mathbf{R} ; V)$ has the weak asymptotic behavior predicted in point (c) of the theorem. We finish the proof with the uniqueness part.

Step 1. Existence of a solution of (2-1) satisfying the maximum principle. Assume that the incoming radiative intensity $\varphi$ satisfies:

$$
B_{\nu}\left(\theta_{m}\right) \leq \varphi(\mu, \nu) \leq B_{\nu}\left(\theta_{M}\right), \quad \text { for a.e. } \mu \text { and } \nu .
$$

Then we define a sequence $\left(f_{n}, T_{n}\right)$ by the following iterative scheme:

$$
\begin{aligned}
& f_{0}=B_{\nu}\left(\theta_{M}\right), \\
& T_{n}=T_{f_{n}}, \quad \text { for } n \geq 0: \\
& \text { for } n \geq 0, f_{n+1} \text { is the unique bounded solution of } \\
& \quad \mu \partial_{x} f_{n+1}+\sigma(\nu) f_{n+1}=\sigma(\nu) B_{\nu}\left(T_{n}\right), \\
& \left.\quad f_{n+1}(0, \mu, \nu)=\varphi(\mu, \nu), \quad \mu \in\right] 0 ; 1[, \nu>0 .
\end{aligned}
$$

By using the maximum principle for the linear transport equation defining $f_{n+1}$ in terms of $T_{n}$, we obtain, after a straightforward induction:

$$
\begin{gathered}
B_{\nu}\left(\theta_{M}\right)=f_{0} \geq f_{1} \geq \cdots \geq f_{n} \geq f_{n+1} \geq \cdots \geq B_{\nu}\left(\theta_{m}\right) ; \\
\theta_{M}=T_{0} \geq T_{1} \geq \cdots \geq T_{n} \geq T_{n+1} \geq \cdots \geq \theta_{m} .
\end{gathered}
$$

Therefore, $f=\lim _{n \rightarrow \infty} f_{n}$ and $T=\lim _{n \rightarrow \infty} T_{n}$ are a solution of (2-1) in the sense of distributions; moreover, in view of (A2), we have

$$
B_{\nu}\left(\theta_{m}\right) \leq f \leq B_{\nu}\left(\theta_{M}\right) \text { and } \theta_{m} \leq T \leq \theta_{M}, \quad \text { for a.e. } x, \mu, \nu .
$$

Step 2. Weak aymptotic behavior for a solution of $(2-1)$ in $L^{\infty}\left(\mathbf{R}^{+} ; V\right)$. We begin with the following lemma:

Lemma. Let $\lambda>0$ and $g=g(x) \in L^{\infty}\left(\mathbf{R}^{+}\right)^{+}$. Assume that $\psi \in L^{\infty}(] 0 ; 1[)^{+}$. Let $u$ be the unique bounded solution of

$$
\begin{gathered}
\mu \partial_{x} u+\lambda u=\lambda g(x), \quad \text { for } x>0, \mu \in[-1 ; 1] ; \\
u(0, \mu)=\psi(\mu), \quad \text { for } \mu \in] 0 ; 1[.
\end{gathered}
$$

Then $\varliminf_{x \rightarrow \infty} u(x, \mu) \geq \varliminf_{x \rightarrow \infty} g(x)$ for a.e. $\mu$.

ProOF OF THE LEMMA. We can write, for $x>0$ and $\mu>0$ :

$$
\begin{aligned}
u(x, \mu) & =\psi(\mu) e^{-\lambda x / \mu}+\int_{0}^{x} \frac{\lambda}{\mu} e^{-\lambda(x-s) / \mu} g(s) d s \\
& =\psi(\mu) e^{-\lambda x / \mu}+\int_{0}^{\infty}\left(\frac{\lambda}{\mu}\right) e^{-\lambda t / \mu}\left[g(x-t) 1_{\{u \leq x\}}\right] d t
\end{aligned}
$$


and

$$
\begin{aligned}
u(x,-\mu) & =\int_{x}^{\infty} \frac{\lambda}{\mu} e^{-\lambda(s-x) / \mu} g(s) d s \\
& =\int_{0}^{\infty} \frac{\lambda}{\mu} e^{-\lambda t / \mu} g(x+t) d t .
\end{aligned}
$$

The announced result follows from Fatou's lemma.

Now let $(f, T)$ be a solution of $(2-1)$ in $L^{\infty}\left(\mathbf{R}^{+} ; V\right) \times L^{\infty}\left(\mathbf{R}^{+}\right)$. We define

$$
v(\mu, \nu)=\varliminf_{x \rightarrow \infty} f(x, \mu, \nu) \text { and } \alpha=\varliminf_{x \rightarrow \infty} T(x) .
$$

According to the above lemma, we have

$$
v(\mu, \nu) \geq B_{\nu}(\alpha) \text { a.e. in } \mu .
$$

Since $f \rightarrow T_{f}$ is nondecreasing (cf. Lemma 1), we deduce from (A6)

$$
T_{v} \geq \alpha
$$

Let $\theta$ be defined by

$$
G(T)=\left\langle\left\langle\sigma(\nu) B_{\nu}(T)\right\rangle\right\rangle .
$$

$G$ is increasing and continuous and one-to-one on $\mathbf{R}^{+}$. Since, by definition, we have

$$
T_{f}=G^{-1}(\langle\langle\sigma(\nu) f\rangle\rangle),
$$

by using Fatou's lemma again, we obtain

$$
\alpha \geq T_{v}
$$

Therefore, $T_{v}=\alpha$, and from (A6) we deduce that $v=B_{\nu}(\alpha)$. The remaining details are routine.

Step 3. Uniqueness. Let $f$ and $g$ be two solutions of $(2-5)$ in $L^{\infty}\left(\mathbf{R}^{+} ; V\right)$. We thus can write

$$
\begin{aligned}
d_{x}\left\langle\left\langle\mu(f-g)^{+}\right\rangle\right\rangle & +\left\langle\left\langle\sigma(\nu)(f-g) \Psi_{f, g}\right\rangle\right\rangle \\
& +\left\langle\left\langle\sigma(\nu)\left[B_{\nu}\left(T_{f}\right)-B_{\nu}\left(T_{g}\right)\right]\left(-\Psi_{f, g}\right)\right\rangle\right\rangle=0 .
\end{aligned}
$$

Therefore, according to Lemma $5,\left\langle\left\langle\mu(f-g)^{+}\right\rangle\right\rangle$is a nonincreasing function of $x$; from the boundary condition, $\left.(f-g)\right|_{x=0}=0$ if $\mu>0$. Thus, $\left\langle\left\langle\mu(f-g)^{+}\right\rangle\right\rangle(x) \leq 0$. According to the previous step, there exists a sequence $x_{n}$ such that

$$
g(x)_{n} \rightarrow B_{\nu}(\beta) \text { for some } \beta \text {. }
$$

From the beginning of the proof of Theorem 4, we know that $\left[\mu\left(g-B_{\nu}(\beta)\right)^{+}\right]$ $\geq 0$. Thus, $\left\langle\left\langle\mu(f-g)^{+}\right\rangle\right\rangle(x)=0$. We deduce from this the following facts:

$$
\left.(f-g)\right|_{x=0}=0 ; \quad\left[B_{\nu}\left(T_{f}\right)-B_{\nu}\left(T_{g}\right)\right]\left(-\Psi_{f, g}\right)=0, \quad \text { a.e. in } x, \nu .
$$

We multiply the equation satisfied by $f-g$ by $\mu / \sigma$; after integration with respect to $\mu$ and $\nu$, we have

$$
d_{x}\left\langle\left\langle\left(\mu^{2} / \sigma\right)(f-g)^{+}\right\rangle\right\rangle+\left\langle\left\langle\mu(f-g)^{+}\right\rangle\right\rangle+\left\langle\left\langle\mu\left[B_{\nu}\left(T_{f}\right)-B_{\nu}\left(T_{g}\right)\right]\left(-\Psi_{f, g}\right)\right\rangle\right\rangle=0 ;
$$

i.e. $\left\langle\left\langle\left(\mu^{2} / \sigma\right)(f-g)^{+}\right\rangle\right\rangle=C$ (where $C$ is a constant). From (A10) we obtain $C=0$. Therefore, $f=g$ a.e. in $x, \mu, \nu$. 


\section{REFERENCES}

1. C. Bardos, R. Caflisch, and B. Nicolaenko, The Milne and Kramer problems for the Boltzmann equation of a hard sphere gas, Comm. Pure Appl. Math. 39 (1986), 323-352.

2. C. Bardos, F. Golse, and B. Perthame, The Rosseland approximation for the radiative transfer equation, Comm. Pure Appl. Math. (to appear).

3. C. Bardos, R. Santos, and R. Sentis, Diffusion approximation and computation of the critical size of a transport operator, Trans. Amer. Math. Soc. 284 (1984), 617-649.

4. P. Benilan, Thèse, Orsay, 1972.

5. A. Bensoussan, J.-L. Lions, and G. Papanicolaou, Boundary layers and homogenization of transport processes, J. Publ. RIMS Kyoto Univ. 15 (1979), 53-157.

6. R. Caflisch, Asymptotic expansions of solutions for the Boltzmann equation, preprint.

7. __ Planar boundary values for rarefied gases, présenté au 17th Biennal Fluid Dynamics Symposium, Odansk, September 1985.

8. 71-109.

9. K. Case and P. Zweifel, Linear transport theory, Addison-Wesley.

10. S. Chandrasekhar, Radiative transfer, Dover, 1960.

11. R. Dautray and J.-L. Lions, Analyse mathématique et calcul numérique pour les sciences et les techniques, tome 3, Masson, 1985.

12. H. Frisch and M. Faurobert, Boundary layers conditions for the transport of radiation in stars, Astronom. and Astrophys. 140 (1984), 57-66.

13. F. Golse, Boundary layers computations arising in radiative transfer with Thomson scattering, preprint.

14. F. Golse and B. Perthame, Generalized solutions of the radiative transfer equations in a singular case, preprint CEA n ${ }^{\circ}$ 2451, Juillet 1985; Comm. Math. Phys. 106 (1986), 211-239.

15. V. Kourganov, Basic methods in radiative transfer, Dover, 1963.

16. E. Larsen, G. Pomranning, and V. Badham, Asymptotic analysis of radiative transfer problems, J. Quant. Spectrosc. Radiative Transfer 29 (1983), 285-310.

17. B. Mercier, Application of accretive operator theory to the radiative transfer equations, SIAM J. Math. Anal. 18 (1987), 393-408.

18. R. Sentis, Equation de Riccati non classique liée aux équations de transport, Nonlinear Partial Differential Equations, Proc. Séminaire du Collège de France, T.V, H. Brezis et J.-L. Lions, Eds., Pitman, 1983.

19. ___ Half-space problems for frequency dependent transport equations. Application to the Rosseland approximation, Proceedings of the meeting "Les mathématiques de la cinétique des gaz" (Paris, June 1985) (to appear).

Centre de mathématiques Appliquées, Ecole Normale Supérieure, 45 rue D'Ulm, 75230 PARIS CEDEX 05, FranCE 